Classification

Physics Abstracts

$61.80 \mathrm{~J}$

\title{
ION IMPACT ON FIELD EMITTER CRYSTALS
}

\author{
J. Y. CAVAILLE and M. DRECHSLER \\ Laboratoire des Mécanismes de la Croissance Cristalline du C.N.R.S., \\ Université Aix Marseille III, Saint-Jérome, Marseille, France
}

(Reçu le 15 mars 1977, accepté le 28 juin 1977)

\begin{abstract}
Résumé. - On fait l'hypothèse que l'impact des ions sur la surface d'un cristal est anisotrope dans le cas où le cristal est un émetteur de champ et les ions sont produits par la collision des électrons émis avec le gaz résiduel. Cette anisotropie est le résultat du fait que presque tous les ions qui bombardent l'hémisphère de la pointe sont produits très près de la surface où le courant électronique est très anisotrope. Les distributions géométriques et énergétiques de l'impact des ions (et les courants absolus) sont déterminées sur la base des trajectoires électroniques et ioniques de Vernickel et Welter. La distribution géométrique forme une image similaire à l'image du microscope électronique de champ. Cette image doit être visible par l'observation des défauts de bombardement dans un microscope à émission de champ. Des expériences préliminaires ont été faites sur l'impact des ions d'hydrogène sur le tungstène. Les résultats ainsi qu'une interprétation des images connues semblent confirmer l'hypothèse d'anisotropie et l'existence d'une image de l'impact ionique.
\end{abstract}

\begin{abstract}
The hypothesis is introduced that the ions impact distribution on a crystal surface is anisotropic, in the case where the crystal is a field emitter tip, and the ions are produced by field electrons collisions with the residual gas. This anisotropy is a result of the fact that nearly all the ions which bombard the tip hemisphere are produced very near the surface, where the electron current is highly anisotropic. The energetical and geometrical distributions of the bombarding ions (as well as absolute ion currents) are determined theoretically on the basis of electron and ion trajectories calculated by Vernickel and Welter. The geometrical ion distribution at the surface should form an image similar to the field electron microscope image. This image should be visualisable by an observation of the bombardment defects in a field emission microscope. Preliminary experiments have been made with a tungsten tip bombarded by hydrogen ions. These experiments as well as an evaluation of known field emission micrographs seems to confirm the anisotropy hypothesis and indicate the existence of the ion impact image.
\end{abstract}

1. Introduction. - Electrons which are emitted from the surface of a cathode into a vacuum gas, produce ions which bombard the emitter surface $[1,3$, $8,10]$. So far it is assumed that the surface of a field emitter crystal is bombarded by ions which have an isotropic statistical distribution. However this seems to be only a rough approximation. The ion density should be specific for each face of the single crystal field emitter (bombardment anisotropy), as described in the first part of this paper. In the second part, the theoretical results are compared with results of preliminary experiments [2], and the ion impact on field emitter crystal is discussed in general.

The study may be interest for several applications. The ion impact on field emitters is sometimes directly applicated as for tip sharpening $[1,20]$ or for the study of ion impact damage. But more often it is an unwanted phenomenon. To avoid it better as at present it may be of interest to study it in more details. This concerns applications as:

(1) field electron guns, where in order to avoid the ion impact special vacuum chambers and U.H.V. pumps must be used in order to obtain sufficient constant currents and long life times.
(2) The initiation of discharges in vacuum. On negative electrodes microprotrusions (tips) can grow which emit field electrons. The ion bombardment thus produced may accelerate the occurence of a breakdown.

(3) In the domain of the production of films by cathode sputtering the cathode surface is under certain conditions transformed in an unwanted arrangement of very many conical tips. This is probably influenced by the impact of ions produced by field electrons emitted from these tips.

(4) The study of gas - solid interactions by field electron microscope techniques is limited by the impact of ions produced by collisions with emitted electrons.

2. Determination of the ion bombardment distribution of the tip crystal. - Electrons which are emitted from a point of a tip follow in the vacuum approximately a field line and ionize on their way gas molecules. The ions which arrive in one point of the tip surface are produced in a well defined geometrical curve (Vernickel and Welter [3]) as indicated in figure 1. In order to determine the distribution of the ion impact 


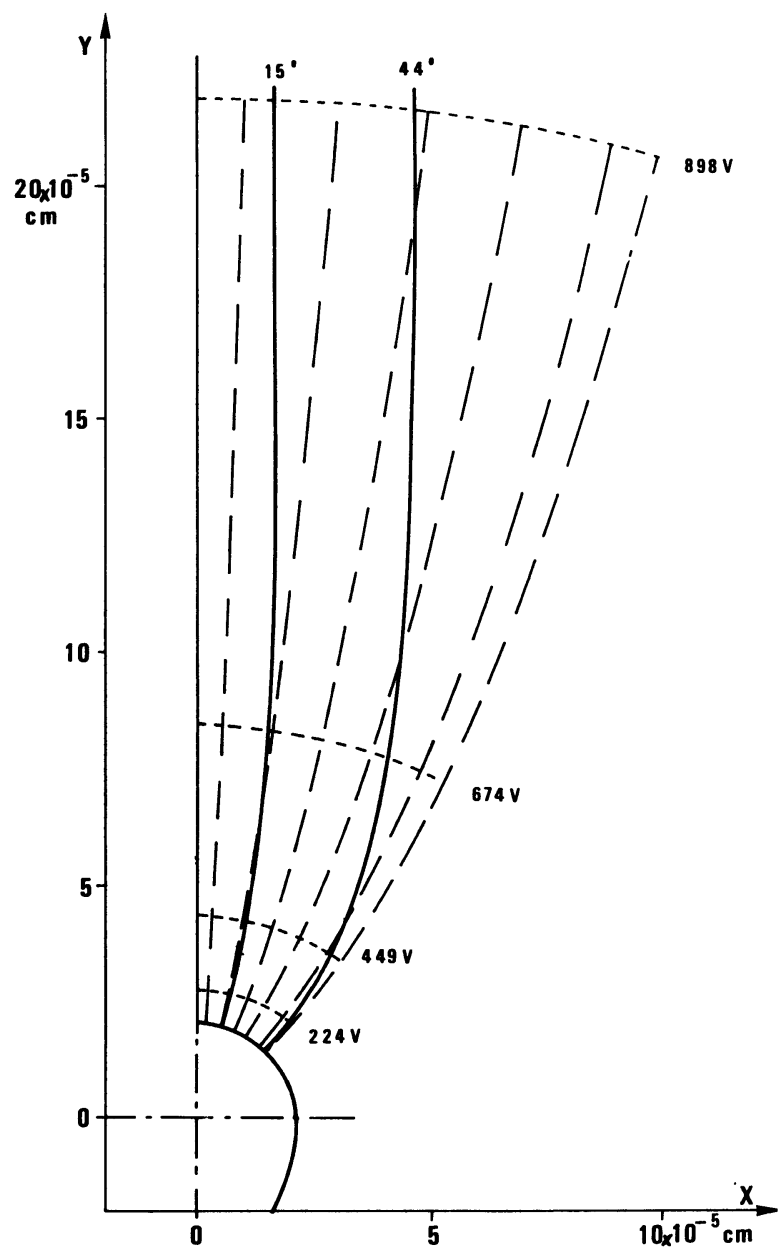

a)

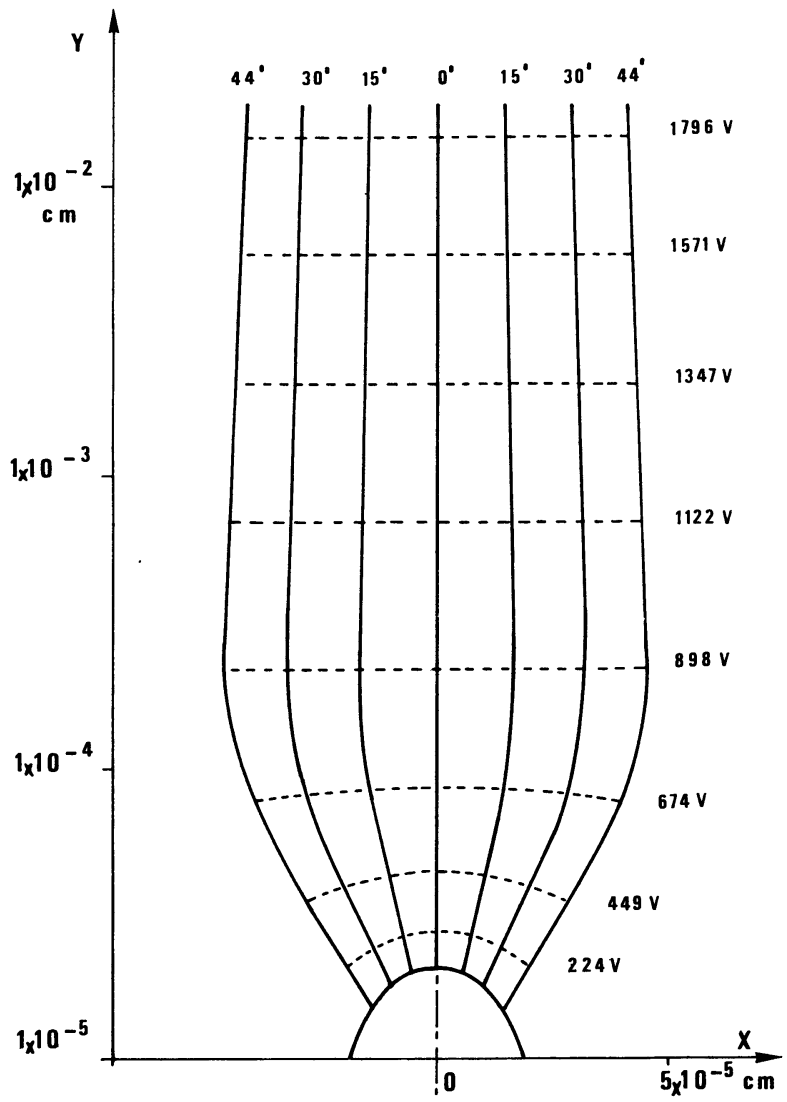

b)

FIG. 1. - Electric field (broken lines), equal potential (small dash lines) and the geometrical locus (solid lines) of the production of those ions which arrive in one point of the surface (after Vernickel and Welter [3]). Calculation for a tip of $2000 \AA$ radius and a form factor [5] 0.2 approximately. Linear ordinate (Fig. $1 a$ ) and logarithmic ordinate (Fig. $1 b$ ).

points at the surface of the tip, essentially three phenomena must be regarded:

(1) The divergence of electrons and ions trajectories (Figs. 2b and c).

(2) The local differences between an electron emission point and the corresponding ion impact points (Fig. 2a).

(3) The anisotropy of the ion impact distribution due to the anisotropy of the field electron emission.

2. 1. DIVERGENCE OF ELECTRON AND ION TRAJECTORIES. - The assumption that electrons and ions emitted from one point follow exactly one trajectory is only an approximation. These particles do not start with energy zero. As a consequence of tangential velocity components the electrons or ions which start from one point must form a fine cone (Figs. 2b and 2c). This corresponds to the limitation of the resolution of the field emission microscopes [1].

Electron trajectories. - In a field electron microscope, the image of an individual atom (in the rare

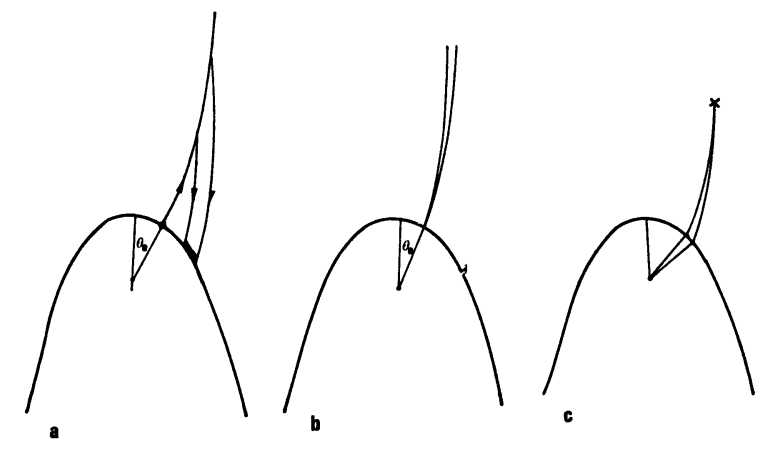

FIG. 2. - Scheme of ion bombardment mechanism.

a) The ion image of an electron emitting point is a radial segment. b) The electrons emitted from one point form a fine cone. c) The ions produced in one point in vacuum arrive as a small spot on the tip surface.

cases, where atoms are visible individually, for instance adatoms of $\mathrm{Cs}$ on $\mathrm{W}$ has a spot diameter in the order of $0.5 \mathrm{~mm}$ (tip radius $1000 \AA$; image diameter 
$5 \mathrm{~cm}$ ), i.e. the spot diameter is roughly 10 times greater than the ideal resolution spot diameter.

The ions which are produced near the microscope screen arrive on the tip in a statistical distribution. However, the ions which arrive on the visible part of the tip surface are produced very close to the tip, i.e. practically within a distance of $10^{2}$ tip radii (see also table I). In the region of maximum ionization, (distance between 5 to 10 radii from the tip) the electron cone diameter is in the order of $100 \AA$ (assuming a linear variation of the cone diameter with the distance from the tip).

\section{TABLE I}

Relative number of ions $\left(\Delta J_{1} / J_{1}\right)$ arriving on the visible part of the tip surface for different intervals of distance $y$ (in radius $r_{0}$ unit) from the tip surface.

$\begin{array}{cl}y & \Delta J_{\mathrm{I}} / J_{\mathrm{I}} \\ 0-10 r_{\mathrm{o}} & -\overline{8} \\ 10-10^{2} r_{\mathrm{o}} & 0.18 \\ 10^{2}-10^{3} r_{\mathrm{o}} & 0.02 \\ 10^{3}-10^{4} r_{\mathrm{o}} & 0.002 \\ 10^{4}-10^{5} r_{\mathrm{o}} & 0.0002 \\ \Sigma \Delta J_{\mathrm{I}} / J_{\mathrm{I}} & 1.00\end{array}$

Ion trajectories. - The ions produced in one point of the vacuum do not arrive in one point of the tip surface, but in a spot of a certain diameter (Fig. 2c). But the ion divergence is less important than the electron divergence; for the same reason the resolution of the field ion microscope [4] is better than that of the field electron microscope, even at room temperature. Here, the ion trajectory divergence is roughly considered by a $30 \%$ increase of the electron cone diameter.

In the case of a very fine tip (radius $400 \AA$ ) the same type of calculation shows that the ions corresponding to one emitting point arrive at the surface within a spot of about $15 \AA$ diameter (instead of $130 \AA$ ).

In conclusion, the electron and ion divergences play only a minor role in the ions bombardment anisotropy (see also paragraph 2. 3.).

Note: the mean energy of the impinging ions is small compared with the voltage $\left(U_{\mathrm{A}}\right)$ between tip and screen. If $U_{\mathrm{A}}: 4000 \mathrm{~V}$, the mean energy is only in the order of $600 \mathrm{eV}$ for a $2000 \AA$ radius tip.

\section{2. Angular distance between electron emission} points and impact points of the corresponding ions. The difference between the electron and the corresponding ion trajectories leads to angular distances (which are called here radial displacements) between an electron emission point and the corresponding ion impact points. These displacements are radial to the tip apex because of the revolution symmetry. These impact points are here called image of the emission point. The radial displacements are calculated for two tips of typical size (400 $\AA$ and $2000 \AA$ radius, corresponding to the radii used by Vernickel and Welter) firstly without consideration of the divergence effect. As a result figure 3 shows the ion impact density distribution for the $400 \AA$ radius tip. So for an emission point distant $5^{\circ}$ from the apex the density of the ions bombardment image has a maximum roughly at $6^{\circ}$ i.e. very near the emission point. However, for an emission point at $30^{\circ}$, this displacement is about $4^{\circ}$. Moreover the width of the distribution increases with the increasing angular distance of the emission point.

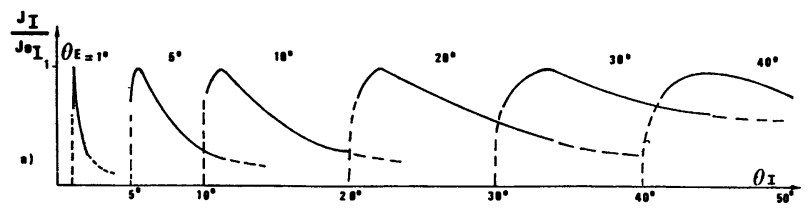

FIG.3a. - Radial angular distribution of the impinging ion density produced by the electrons emitted from several points of different angular distance $\left(\theta_{E}\right)$ from the tip apex.

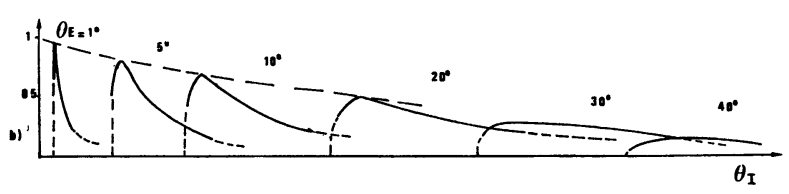

FIG. $3 b$. - Same distribution but with consideration of the variation of the electric field along the surface [5] (mean work function $\left.4.5 \mathrm{eV}, F_{\text {apex }}=4 \times 10^{7} \mathrm{~V} / \mathrm{cm}\right)$.

In the case of the $2000 \AA$ tip, angular displacement are similar but the distributions are slightly larger (see Fig. 5).

If the decrease of the electric field with the angular distance from the tip apex is considered [5] (and a mean work function of $4.5 \mathrm{eV}$ assumed) one obtains distributions as show, in figure $3 \mathrm{~b}$. Within one distribution there is always a correlation between the position of the impact point and the ion energy $(E)$. One example is shown in figure 4a. Each distribution starts at energy $O$ and arrives its maximum at an energy which is considerably smaller than the energy corresponding to the tip-screen voltage $(4000 \mathrm{~V})$. The general numerical result is shown in figure $4 \mathrm{~b}$ : the angular position of each ion impact point as a function of the angular position of each electron emission point with the ion energy as parameter. Consequently, the energy and relative ion impact density can be determined for each point of the emitter surface. The influence of electron and ion trajectories divergence is shown in figure 5. The divergences lengthen the radial distributions only slightly. There exists also an divergence normal to the radial direction. Therefore, the ion bombardment image of one emitting point is not a line, but a somewhat broaden line or a longuish elliptic spot. In fact, as the divergence increases with ion energy, (or distance from the 


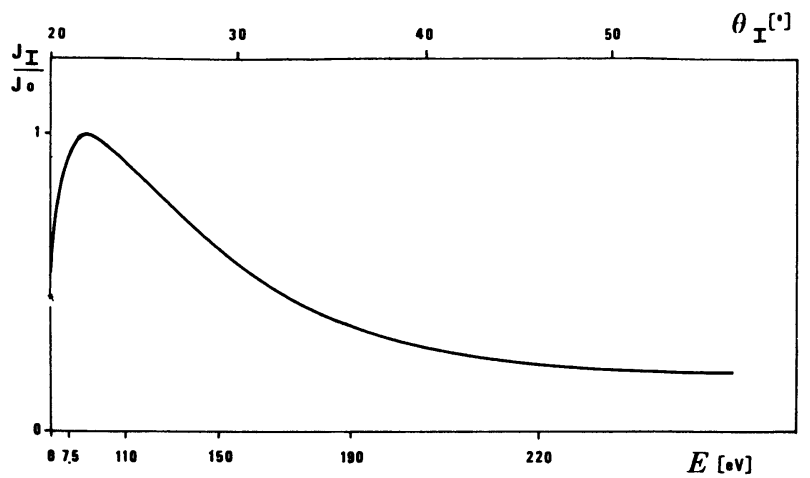

Fig. 4a. - Typical energetical (and angular) distribution of impinging ions. Example corresponding to the distribution $\theta_{\mathrm{E}}=20^{\circ}$ in figure $3 \mathrm{a}$.

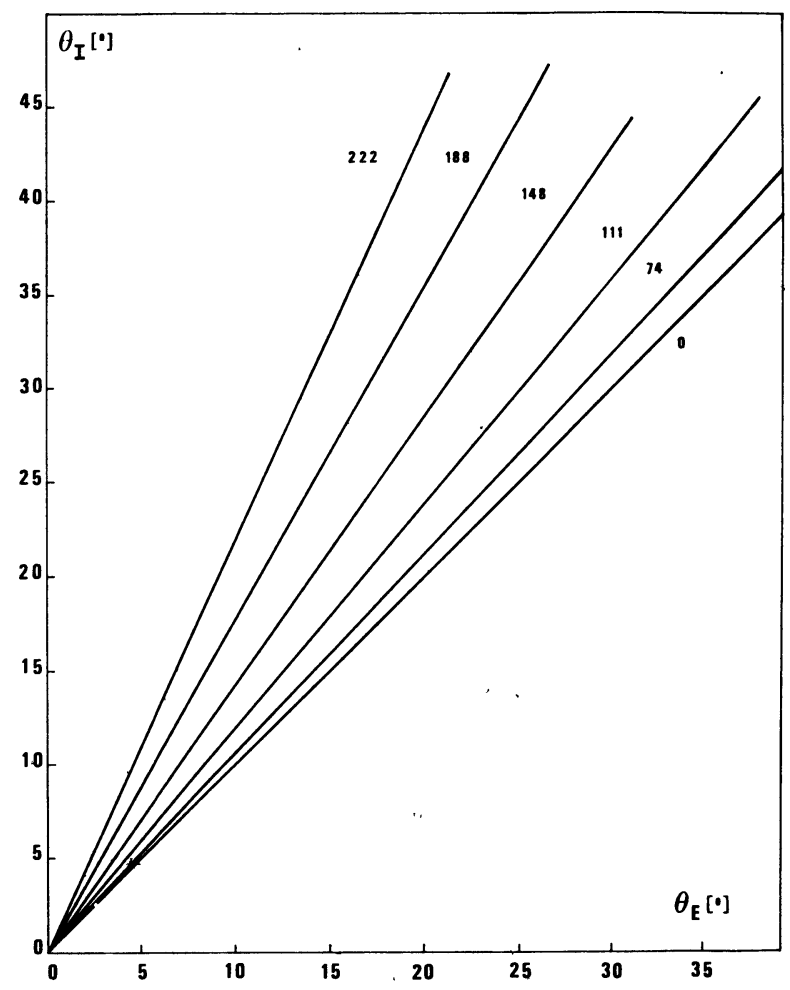

FIG. 4b: - Angular position $\left(\theta_{1}\right)$ of the ion impact point as a function of the angular position $\left(\theta_{\mathrm{E}}\right)$ of the electron emission point, for typical ion energies in $\mathrm{eV}$ ( $400 \AA$ radius tip).

tip surface) the spot is not exactly elliptic but should have the form of a longuish drop.

The influence of the ionization distance on one hand on the trajectory divergence and on the other hand on the radial displacement is shown in figure 6.

This is a projection of the emitting part of the tip crystal (broken line). The centers of some faces are indicated. Two electron emission points are considered: on the bottom of the figure one point $\left(8^{\circ}\right.$ distant from the opex) and on the top another point $\left(18^{\circ}\right.$ distant from the apex which corresponds to the emission of the (012) face in the case of a (110) oriented bcc crystal). The circles represent impact spots of

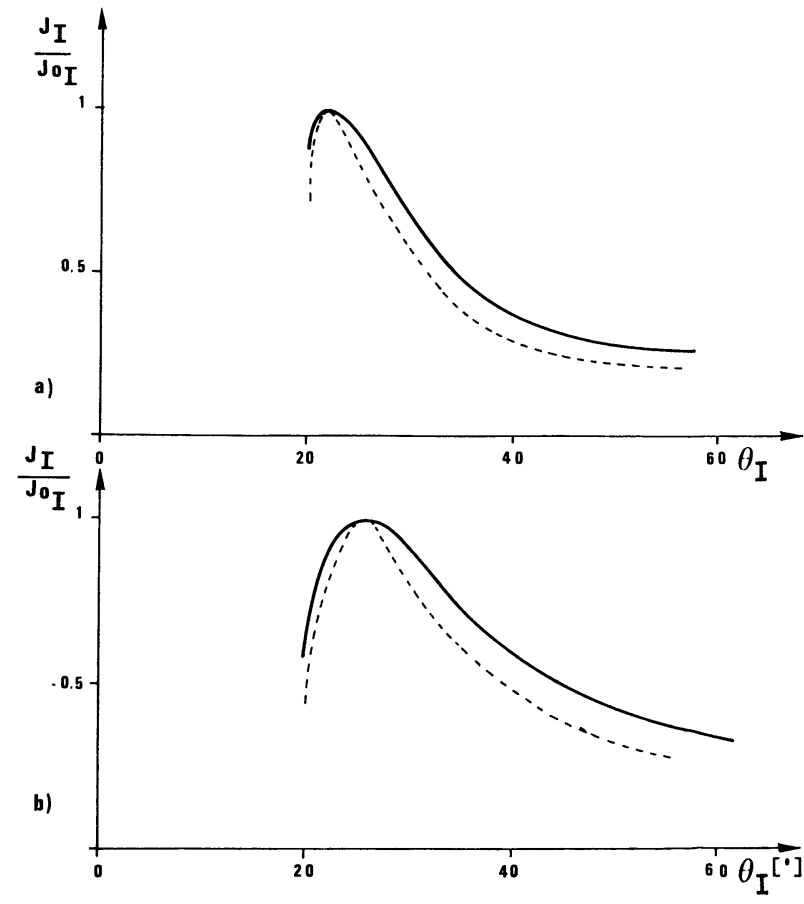

FIG. 5. - Influence of trajectory divergence on the radial angular distribution of ion impacts. Radial angular ion density distribution without divergence (broken line) and with divergence (solid line) for ions produced by a $20^{\circ}$ electron emission point. Calculation for a $400 \AA$ radius tip (Fig. 5a) and a $2000 \AA$ radius tip (Fig. 5b).

those ions which are produced at special distances (and which have special energies). The numbers on the circles indicate the ionisation distance from the tip surface in tip radius $\left(r_{\mathrm{o}}\right)$ unit. The numbers in parenthesis indicate the relative number of ions impinging within the spot. The result presented in this figure show that ions of relative high density arrive at the surface always in fairly small spots. But ions produced more far away from the tip ( $\left.>10^{2} r_{0}\right)$ have a much smaller density and arrive at the surface as a greater spot. For long ionization distances ( $>\simeq 3 \times 10^{2} r_{\mathrm{o}}$ ), the ions produced by one electron emission point not too far from the apex cover more than the whole emitting surface (circle of which only a part is visible on top of figure 6), i.e. arrive in statistic manner but with extreme low density. Of course, the ions which arrive at the visible part of the tip are produced only in the region near the tip axis.

In conclusion, electron and ion trajectory divergences are not important enough to produce an ion bombardment in a statistic manner.

2. 3. ANISOTROPY OF ION IMPACT. - In the preceding part of this paper, constant work function was assumed. In reality, the work function is different for each face. Electron emission varies strongly with the work function (Fowler-Nordheim equation) as well as with the local field strength (emission from protrusions). The strong electron emission anisotropy must provoke significant ion impact anisotropy. To 


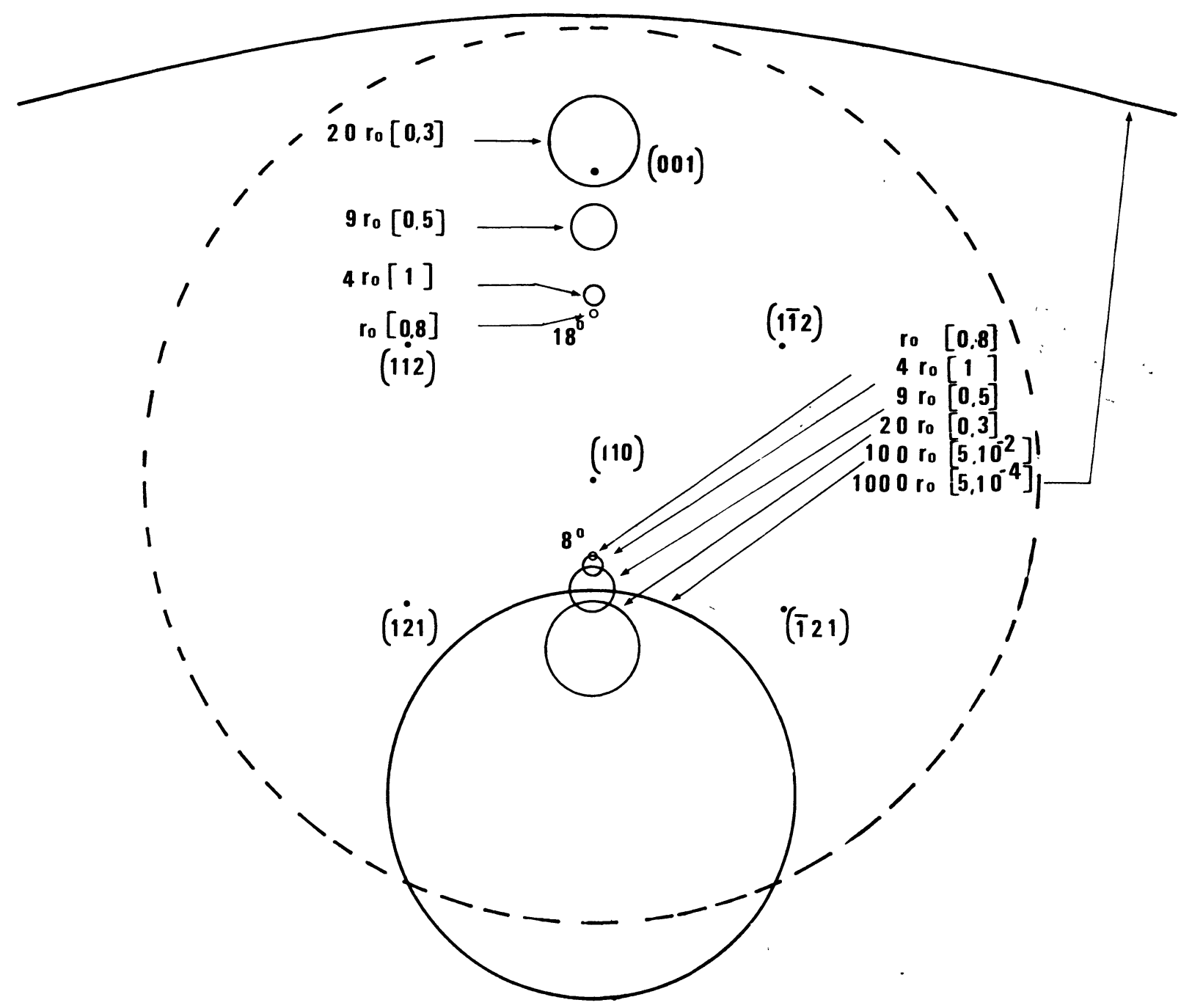

Fig. 6. - Simplified presentation of the simultaneous action of trajectory divergence and the angular distance between emission and impact points (details in the text).

determine the ion anisotropy, the work function anisotropy must be known.

In the following, two cases are considered in a simplified manner, (1) tungsten (or another bcc crystal) / rare gas, and (2) tungsten / hydrogen.

In the first case no adsorption occurs at room temperature so that the work function anisotropy is that of the clean tungsten crystal (if work function changes by ion impact defects are neglected). Simplified ion impact images are determined from the radial distributions (Fig. 3) and shown in figure 7, together with the corresponding electron image.

In the figure $7 \mathrm{~b}$ and $\mathrm{d}$ the regions of high electron emission and high ion impact density are outlined.

The ion bombardment image should look like a slightly radial magnified electron image but must be more blurred. Furtheron with increasing angular distance from the tip apex (image center) the mean field electron density decreases while (the mean ion energy increases and) the mean ion impact density may be roughly constant. The latter should be a consequence of the asymmetrical distribution tails which may compensate the decrease of the distribution maxima with increasing angular distance (Fig. 3b).

The field electron density of a low work function face as (012) is roughly $10^{4}$ times (degree of anisotropy) greater than the emission of a high work function face such as (110), if the field has a typical value in the order of $3 \times 10^{7} \mathrm{~V} \cdot \mathrm{cm}^{-1}$. The degree of anisotropy of the ion bombardment should have nearly the same order of magnitude, as the degree of field electron anisotropy. The face of the lowest ion density must be the central (011) face (Fig. 7a), while the density on the $\{110\}$ faces of $60^{\circ}$ distance from the apex should be not so low as a consequence of the trajectory divergence (Fig. 6) and the radial displacement.

The case hydrogen on tungsten is presented in figure 8. Adsorption occurs immediatly after hydrogen inlet. The image $8 \mathrm{a}$ is typical for an adsorption on 


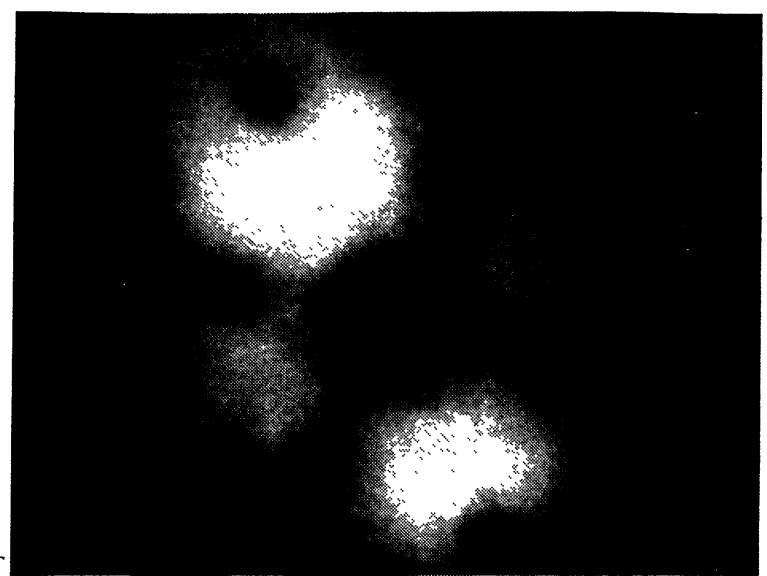

a)

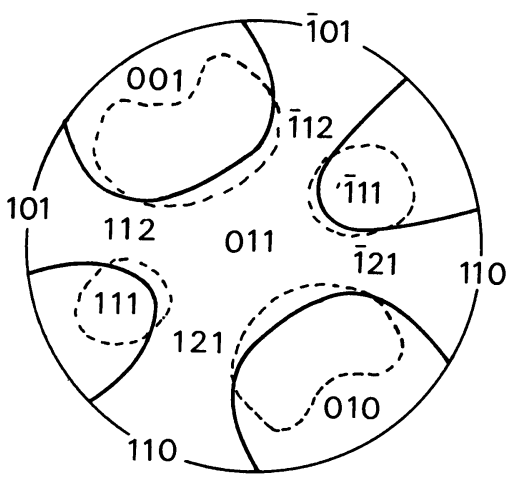

b)

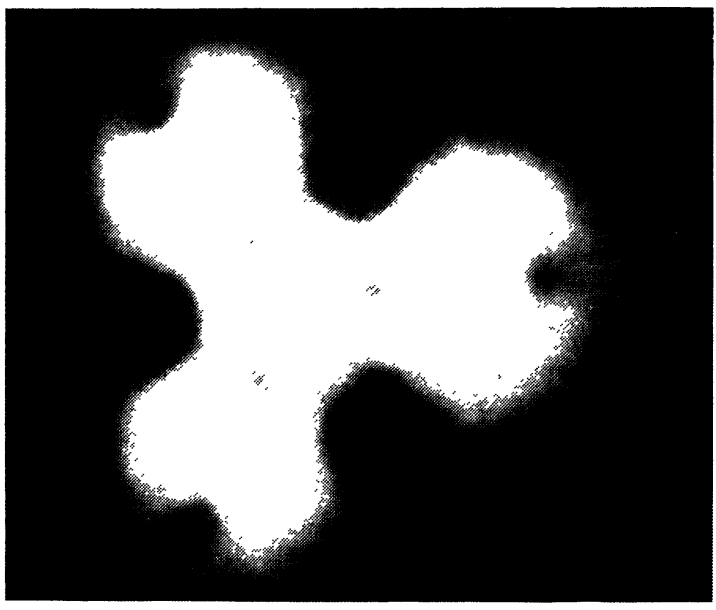

c)

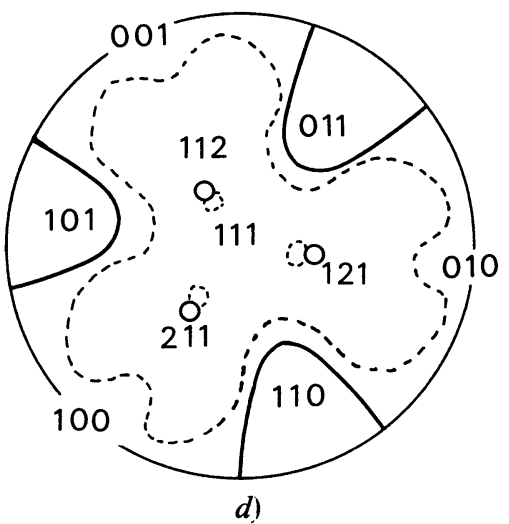

d)

Fig. 7. - Ion bombardment anisotropy of a bcc crystal (tungsten) without adsorption. a) Field electron microscope image, of [110] orientation. $b$ ) Superposition of the electron image (broken lines) and the ion bombardment image (solid lines). c) Microscope image of [111] orientation (S. Ranc [6]). d) Superposition analogous to figure $b$.

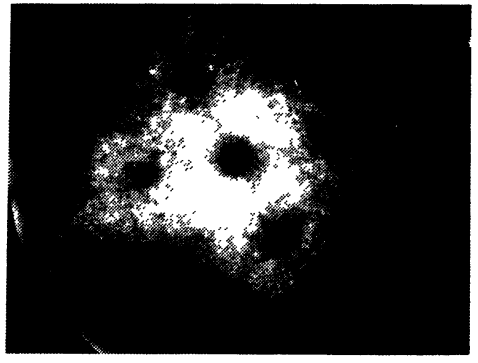

a)

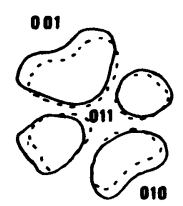

b)

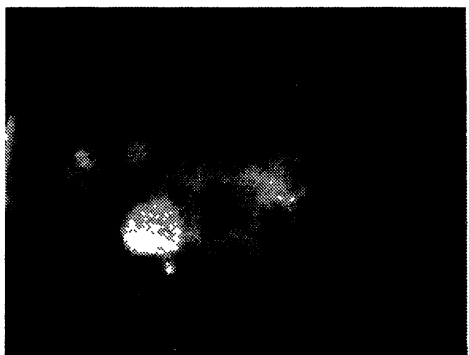

c)

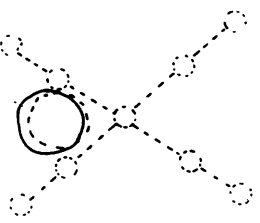

d)

FIG. 8. - Ion bombardment anisotropy with adsorption (hydrogen on tungsten). a) Field electron microscope image, $p-1.5 \times 10^{-2}$ torr hydrogen, $6760 \mathrm{~V}, 5.2 \mu \mathrm{A}$, after 4 minutes bombardment. b) Superposition of electron emission and ion bombardment (solid lines) images. c) Image after beginning of protrusion growth. Same conditions as in figure $8 a$, but after 25 minutes, $8.5 \mu$ A. $d$ ) Superposition of the electron and ion bombardment image of the protrusion. 
a bombarded surface. For a lower hydrogen pressure ( $<10^{-5}$ torr) and only up to some seconds after hydrogen inlet, adsorption images are obtained which correspond to the known typical hydrogen on tungsten images [7]. The figure 8b shows (as Fig. 7b) the superposition of the electron emission and the ion bombardment image. An essential difference compared to the rare gas case (Fig. 7) is the lower value of ratio between maximum and minimum density (degree of anisotropy) which may be of the factor 100 .

An example where electrons are emitted essentially from a protrusion is shown in figure 8c. This protrusion is grown after a long bombardment period (under the influence of the field [2]). The ion impact is very localized in such a case. The estimated ion impact density as a function of the angular distance is shown in figure 9 for crystals of two different orientations and two crystallographic zones.

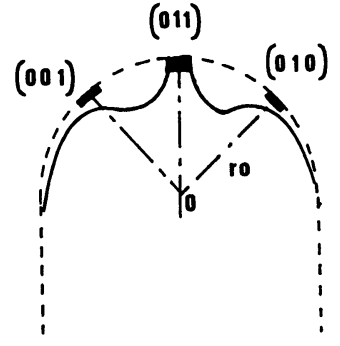

a)

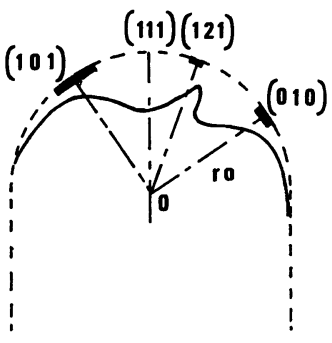

FIG. 9. - Estimated ion impact density as function of the angular distance (solid lines). Presentation in polar coordinates; $r=r_{0}$ corresponds on one hand to density $\mathrm{O}$, and on the other hand to the tip surface (broken line); the amplitude is directed towards zero.

a) Case of figure $8 \mathrm{a}$, zone [100].

b) Case of figure 7c, zone [110].

2. 4. AbSOlUte CURRENTS (ORDER OF MAGNitUde). - Total ion current. - The total number of ions $\left(I_{\mathrm{i}}\right)$ produced by the total field electron current $I_{\mathrm{e}}$ is:

$\Gamma_{\mathrm{i}}=\alpha_{\mathrm{o}} P l I_{\mathrm{e}}$

$\alpha_{0}$ is the mean number of ions produced per electron, $\mathrm{cm}$ and torr, [9],

$p$ is the gas pressure in torr,

$l$ is the mean trajectory length in cm (tip-screen distance).

A typical example is:

$p=10^{-3}$ torr, $I_{\mathrm{e}}=10^{-6} \mathrm{~A}, \alpha_{\mathrm{o}} \simeq 2 \mathrm{~cm}^{-1}$ torr $^{-1}$ (hydrogen, neon),

$l=5 \mathrm{~cm}$, so the total ion current is: $\Gamma_{\mathrm{i}}=10^{-2}$ $I_{\mathrm{e}}=10^{-8} \mathrm{~A}$.

Ion current arriving on the visible part of the surface. - The number of ions $I_{\mathrm{i}}$ which arrive at the visible part of the tip is only a very small fraction of the total ion current. To calculate the order of $I_{\mathrm{i}}$, it is assumed:

(1) the angular distribution of the electron current density is those known from the literature [5] (only mean work function considered),
(2) the electron trajectories in the direct neighbourhood of the tip are straight lines normal to the surface,

(3) the ionization cross sections of the different gases are constant in the energy region from 50 to $1000 \mathrm{eV}$. This approximation is possible as these cross sections have fairly flat maxima in this region [9],

(4) the ions which arrive in the visible part of the tip surface are produced in a cylinder coaxial to the tip [3, 8], (the cylinder radius varies between one and two tip radii, see also Fig. 1b).

The number of ions produced per second, is proportional to the gas pressure and to the field electron current. The ion current $\left(I_{i}\right)$ which arrive on the visible part of the tip surface is obtained by integration of the ions which are produced in the cylinder:

$I_{\mathrm{i}}=\alpha_{\mathrm{o}} A p r_{\mathrm{o}} I_{\mathrm{e}}$

$\alpha_{0}$ is the number of ions produced per electron. $\mathrm{cm}$. torr [8],

$r_{\mathrm{o}}$ is the tip radius in $\mathrm{cm}$.

$A$ is a constant which increases with the form factor [5]:

$3<A<8$.

Typical experimental data are. $-\alpha_{0} \simeq 2$ (hydrogen), $\dot{p}=10^{-3}$ torr, $r_{\mathrm{o}}=2000 \AA, I_{\mathrm{i}} \simeq 10^{-7} I_{\mathrm{e}}$; that means for the impact of one ion, an emission of $10^{7}$ electrons is needed. Note: The current is calculated for constant gas pressure. A rough calculation has shown that the influence of ion pumping is negligible in our experiments, (analogous to results of Vernickel and Welter [3]). Nevertheless, in some extreme cases, (electron current density greater than $10^{5} \mathrm{~A} / \mathrm{cm}^{2}$ and molecules of relatively great mass) near the apex the pressure can be reduced non negligibly. In such a case ion pumping should modify the angular distribution of ion bombardment; in particular, (1) there should be an increase of the displacement between electron emission points and ion impact points, and (2) a decrease of the total number of ions which arrive on the central part of the emitting surface.

The preceding result enables to determine the time after which the number of impinging ions is equal to the number of atoms of a monolayer ( $\simeq 1015$ atoms/ $\mathrm{cm}^{2}$ ). The result is: if $p=10^{-3}$ torr, this time is of the order of one second.

Place of ion production. - As regards the ions which arrive on the visible part of the tip surface it might be of interest to obtain more information on the local distribution of ionization. In particular, is the high ionization probability in the regions near the tip $\left(<10^{2} r_{\mathrm{o}}\right)$ perhaps compensated by the long distance $(\simeq 5 \mathrm{~cm})$ of the whole electron trajectory? The answer is given in the table I. $98 \%$ of all these ions are produced in the region $y<10^{2} r_{\mathrm{o}}$. This corresponds to the fact the mean energy of the ions arriving on the tip surface is very low in comparison 
to the value of the tip-screen voltage. The ion production very close to the tip is also the reason why a change of the anode geometry (which practically does not change the electric field near the tip) does not considerably change the ion impact on the visible part of the tip surface.

\section{Experimental verification (ion impact anisotropy} and radial displacement). - The ion bombardment anisotropy has not yet been studied as far as we know. Here we present some preliminary experimental results which are obtained in the course of a study on predischarges and surface self-diffusion by ion bombardment.

The ion bombardment anisotropy can be visualised only indirectly by field emission microscope observations of the surface defects produced by ion bombardment. The number of these defects must be a function of the number of the impinging ions. Studied is in particular the impact of hydrogen ions on a tungsten tip in a field electron microscope for a hydrogen pressure between $10^{-5}$ and $10^{-2}$ torr. The ion impact appears on the microscope screen as small just visible scintillations.

In the region of the central (011) face no scintillations appear. This is in agreement with the anisotropy calculation (see also Fig. 6). The ion bombardment leads to the growth of tungsten protrusions [2] and this phenomenon depends on the local number of impinging ions. The experiment shows that the protrusions are formed by preference in the (111) region (up to the faces (112), (121) and (100)), but not near the central (011) (see also Fig. 8c). In agreement with the calculation this is a region where relatively high ion bombardment density is predicted. Nevertheless, protrusion growth appears less often in the region around (012) where high ion bombardment density is also predicted. This can be explained by the assumption that the surface self-diffusion which is necessary for the formation of protrusions, is smaller around (012).

The protrusions around (111) (left side of Fig. 8c) are obviously greater than the ones around (111) (right side of this figure). This is a further indication that this growth depends on the value of the field strengh. In fact the field strengh around (111) is slightly greater than around (111), as a consequence of the fact that the 011 axis of the tip crystal is very slightly inclined versus the tip axis. Such an inclination must exist because the left side of the crystal image (Fig. 8a and c) is slightly brighter than the right side, which was already visible on the image of the clean crystal.

The protrusions are removed if the tip is heated in the absence of the field to a temperature $(T>$ $1200 \mathrm{~K}$ ) where thermal surface self-diffusion occurs. This is a proof that the protrusions are tungsten protrusions.
One protrusion is normally dominant (Fig. 8c). This result is in agreement with our hypothesis on the growth mecanism [2]. The speed of protrusion growth should increase drastically during growth because: the field strengh at the protrusion apex increases during the growth which leads to an important increase of the electron emission and the ion impact in the neighbourhood of the protrusion.

In our experiments the breakdown is avoided by the use of a relay [2] which cuts the high voltage if the current passes a certain value. Then, after a regeneration of the tip by a heat treatment the tip is used for a new experiment.

In the field electron image of a bombarded surface, rarely impacts of individual ions (small bright spots) are found also in regions of small electron density as (112) for instance. This result can be explained as a sign of the rare impact of ions produced at relatively great distance (statistic impacts) from the tip surface (see Fig. 6).

The theory predicts a radial displacement of the ion impact image in comparison to the field electron image. This can be qualitatively controled by interpreting field electron micrographs of Vernickel [10] on the bombardment of $\mathrm{W}$ by $\mathrm{Ar}^{+}$. On clean $\mathrm{W}$ maximum emission occurs between $20^{\circ}$ to $30^{\circ}$ apex distance ((012), (013), (122), (133)). On heavy bombarded $\mathrm{W}$ maximum emission occurs between $30^{\circ}$ and $60^{\circ}$ distance. The average displacement found $\left(\simeq 30^{\circ}\right.$ ) seems to be greater than those predicted. However it must be considered, that in the case of heavy $\mathrm{Ar}^{+}$bombardment the image character changes drastically, i.e. many little spots (craters) become visible, which do mask the face specific emission. In such a case the regions of maximum emission should be displaced continiously leading with time to a much greater total displacement. This does qualitatively explain the observation.

In conclusion, preliminary experiments are in agreement with the predicted ion impact anisotropy.

4. Discussion. - 4.1. NATURE AND STRUCTURE OF THE BOMBARDED SURFACE. - Little is known on the precise nature and the structure of ion bombarded surfaces. A discussion of the experimental results permit to obtain a few additional informations in this direction. The hydrogen ions of $10^{2}$ to $10^{3} \mathrm{eV}$ energy penetrate into the crystal bulk for a relatively small depth in the order of $50 \AA$ [15] which is also theoretically predicted [19]. The ions produce certainly many crystal defects in the surface film, a fact which has also been studied by field ion microscopy using seperated ion sources $[16,17,1,18]$. It is astonishing that in the hydrogen-tungsten experiment the tip crystal after the bombardment is still a monocrystal which can be concluded from the figure $8 \mathrm{a}$. The $\{011\}$ and $\{112\}$ faces present (Fig. 8a) local emission minima. The hydrogen within the bulk 
should be incorporated in form of interstitials. It is probable that in course of the bombardment the hydrogen concentration within the bulk of the thin surface film arrives a value where the number of hydrogen atoms is of the order of the number of tungsten atoms. The field electron microscope experiment shows $\left(10^{-3}\right.$ torr hydrogen) that a few seconds after the beginning of the ion impact the image (Fig. 8a) does not change any more, indicating a dynamic equilibrium where the number of impinging hydrogen particles is equal to the number of hydrogen particles leaving the surface.

Normally, hydrogen does practically not diffuse into the tungsten lattice. However these experiments show that hydrogen diffusion must occur in a tungsten lattice, which contains bombardment defects and hydrogen interstitials. The incorporation of the hydrogen in the tungsten bulk provokes certainly a strong tendency for a dilatation of the lattice with a decrease of the tungsten atom density. Consequently also the two-dimensional density of each crystal face should be decreased. Therefore it is surprising that the surface film and the inner tungsten bulk do form still a single crystal (with certainly important atomic tensions). This can be explained by the hypothesis that the bulk of the surface film contains a great number of (more or less periodic) defects as stacking faults and dislocations in a facespecific manner.

The ion impact anisotropy could provoke an anisotropy of the cathode sputtering and consequently an anisotropic tip profile change with a geometry similar to the curves shown in figure 9. However, the field electron image does not show any sign of such a profile change. Obviously, the beginning of the formation of pits at the surface is compensated by an elimination of any type of pits by an ion impact induced surface self-diffusion. This can be regarded as one proof for the existence of this typo of surface self-diffusion [2]. In other words the number of atoms migrating along the surface by this type of diffusion is greater than the number of atoms which are sputtered.

4.2. PossibILITY OF TIP HEATING BY ION BOMBARDMENT. - Does the ion bombardment heat the tip? To answer this question, the ion bombardment heating can be compared with the field electron emission heating $[11,12,13]$ (Nottingham effect). The Nottingham heat is in the order of $0.2 \mathrm{eV} / \mathrm{electron}$. One emitted electron produces $10^{-7}$ ions of less than $10^{3} \mathrm{eV}$ impact energy. Therefore, the heat produced by the ions which are produced by one emitted electron is smaller than $10^{-4} \mathrm{eV}$. Consequently, the heat produced by the ions which arrive on the visible part of the tip surface is 2000 times smaller than the Nottingham heat for the same electron current and it is known that the temperature increase due to the Nottingham heat is negligable under these conditions. The bombardment of the whole cathode (tip, heating loop and connecting wires) should be also considered. If the total ion current is $10^{-8} \mathrm{~A}$ (example after equation (1)), the total bombardment power output is in the order of $10^{-5}$ watt. The heat conductivity of the system is certainly sufficient, that a $10^{-5}$ watt ion heating does not produce a considerable temperature increase. A measurement of the resistance of the heating loop has confirmed this in the case of the hydrogen-tungsten system.

In conclusion, the temperature increase by ion bombardment is negligible in the described experiments.

Acknowledgments. - We thank very much Dr. H. Vernickel of Max-Planck-Institut für Plasmaphysik for reading the manuscript and valuable comments.

\section{References}

[1] Müller E. W., Z. Phys. 106 (1937) 132; Müller E. W., Ergeb. ex. Wiss. XXVII, (1953), 290. Müller E. W. and Tsong T. T., Field Ion Microscopy, (Elsevier, New York), 1969.

[2] Cavaille J. Y. and Drechsler M., send to Surf. Sci.

[3] Vernickel H. and Welter H., 16th Field Emission Symposium, Pittsburg 1969; und Ber. Inst. Plasmaphysik, Garching bei München, JPP, 7/2, 6/88 (1970).

[4] Müller E. W., Z. Phys. 131 (1951) 136.

GoOd R. H. and MülleR E. W., Handb. Phys. XXI (1956) 176.

[5] Drechsler M. and Henkel E., Z. Angew. Phys. 6 (1954) 341.

[6] Ranc S., thèse 1974, Département de la Physique des Matériaux, Université de Lyon, Villeurbanne.

[7] Domke M., Jähning G. and Drechsler M., Surf. Sci. 42 (1974) 389.

[8] Dranova Zh. I., Mikhailovskil I. M., Sov. Phys. Solid State 12 No. 1 (1970) 104.

[9] ARDENNE M. V., Tabellen der Elektronen und Ionenphysik, (Deutscher Verlag der Wissensch) Berlin,1956.

[10] Vernickel H., Ber. $d$ Inst. Plasmaphysik, Garching bei München, IPP 2/72, 1968.
[11] DreChSLER M. and JäHNING G., Z. Naturforsch. 18a (1963) 1367.

[12] Swanson S. W., Crouser L. C. and Charbonnier F. M., Phys. Rev. 151 (1966) 327.

[13] Drechsler M., Rev. Gén. Elect. 80 (1971) 569.

[14] Behrisch R., Böttiger J., Eckstein W., Roth J., ScherZER B.M.U., J. Nucl. Mater. 56 (1975), 365.

[15] Panitz J. A., 23rd Intern. Field Emission Symposium, Pennsylvania State University, August 1976.

[16] Müller E. W., Proc. 4th Intern. Symp. Reactivity of Solids, Amsterdam, ed. J. H. de Boer, (Elsevier), 1960, 682.

[17] Brandon D. G., Wald M., RalPh B., Proc. Intern. Conf. Crystal Lattice defects, Kyoto 1962, J. Phys. Soc. Japan 18 Suppl. II (1963) 324.

[18] Strayer R. W., CoOper E. C., Swanson L. W., 12th and 14th Field Emission Symposium, Pennsylvania State University 1965 and Washington D. C. 1967.

[19] Lindhard J., Scharff M., Schott H. E.; Danske Videnskab Selstab Mat. Fys. Medd. 33 (1963) 14.

[20] Janssen A. P., Jones J. P., J. Phys. D, Appl. Phys. 4 (1971) 118. 